Check for updates

Cite this: RSC Adv., 2017, 7, 56732

Received 2nd November 2017 Accepted 10th December 2017

DOI: 10.1039/c7ra12062a

rsc.li/rsc-advances

\section{Improving osteogenesis of PLGA/HA porous scaffolds based on dual delivery of BMP-2 and IGF- 1 via a polydopamine coating $\dagger$}

Jun Zhang, Jianan Li, Guoliang Jia, Yikun Jiang, Qinyi Liu, Xiaoyu Yang and Su Pan (D)*

To engineer bone tissue, an ideal biodegradable implant should be biocompatible, biodegradable, osteoinductive and osteoconductive. However, the lack of bioactivity has seriously restricted the development of biodegradable implants in bone tissue engineering. In this study, we have developed a three-dimensional porous poly(L-lactic-co-glycolic acid)/hydroxyapatite (PLGA/HA) scaffold based on the dual delivery of bone morphogenic protein 2 (BMP-2) and insulin-like growth factor 1 (IGF-1) via polydopamine (PDA) coating. The aim of this study was to improve the osteoinductivity of biodegradable implants for clinical bone repair via a PDA-assisted BMP-2 and IGF-1 surface modification strategy. Our study demonstrated that PDA layer surface modification could more efficiently immobilize BMP-2 and IGF-1 on the scaffold surfaces than physical adsorption, and the immobilized growth factor was released slowly and steadily from the scaffold in a sustained manner. MC3T3-E1 cell attachment and proliferation on IGF-1 and BMP-2-immobilized porous scaffolds were much higher than other groups. According to an in vitro osteogenesis assay, alkaline phosphatase (ALP) activity, the expression of osteogenesis-related genes and the mineralization capacity of MC3T3-E1 cells were increased by the incorporation of BMP-2 and IGF-1. In vivo, digital radiograph evaluation demonstrated that there was the most rapid healing in the defects treated with the IGF-1 and BMP-2 immobilized porous scaffold compared with the other groups eight weeks after implantation. In vitro and in vivo results of this study revealed that the PDAassisted surface modification method can be a useful tool to graft biomolecules onto biodegradable implants, and the dual release of BMP-2 and IGF-1 could promote cell proliferation and osteogenesis differentiation. Based on the results, the surface modification strategy with growth factors has great potential for the enhancement of osteointegration of biodegradable bone implants.

\section{Introduction}

Modern clinical practice in orthopaedics has confirmed that autogenous bone grafting is the gold standard for the treatment of bone defects. However, many drawbacks have limited its development, such as morbidity of the donor site, immune reaction, nerve damage, transfer of pathogens and limited availability. ${ }^{1}$ These drawbacks have led to the exploration and development of various biomaterials as bone substitutes and grafts for the treatment of bone defects. In the last decade, much attention has been paid to bone substitutes composed of various biomaterials such as ceramics, PLGA, PCL, hydroxyapatite and bioactive glasses. ${ }^{2-4}$ Among these biomaterials, PLGA has attracted much interest because of its excellent biodegradability, mechanical properties, low immunogenicity and toxicity, and adjustable degradation rate through altering the

Department of Orthopedic Surgery, The Second Hospital of Jilin University, Changchun, Jilin 130041, China. E-mail: JilinPansu@163.com

$\dagger$ Electronic supplementary information (ESI) available. See DOI: $10.1039 / \mathrm{c} 7 \mathrm{ra} 12062 \mathrm{a}$ ratio of lactic to glycolic acids. On the other hand, hydroxyapatite $\left(\mathrm{Ca}_{10}\left(\mathrm{PO}_{4}\right)_{6}(\mathrm{OH})_{2}, \mathrm{HA}\right)$, the main component of bone tissue in the human body, has attracted much interest due to its biocompatible, osteoconductive, nontoxic, non-inflammatory, and adequate mechanical properties. $^{5}$ Moreover, $\mathrm{HA}$ is a porous material which allows in growth of capillaries and other vessels; this results in the perfusion of metabolic oxygen and nutrients to cells that lie in the scaffold, as well as cells from the host that integrate into it, which can effectively improve the biological activity of scaffolds. Composites of poly(lactide-co-glycolide) (PLGA) and HA have been developed into orthopaedical materials. In recent years, more and more studies on HA and PLGA nanocomposites for biodegradable bone implants have been reported., ${ }^{2,6}$ However, it is widely acknowledged that ideal bone implants should be biodegradable, biocompatible, osteoinductive, and osteoconductive. PLGA has hydrophobic surfaces that do not promote cell adhesion and proliferation, and HA alone possesses limited osteoinductive ability, which has limited the application of PLGA/HA nanocomposites in bone defect repair. ${ }^{7,8}$ One strategy to solve this problem is to combine the biomaterials with growth factors to 
enhance their bone regeneration performance, which could compensate for the lack of osteointegration of the substrates.

Numerous growth factors are known to induce osteogenic differentiation and promote bone regeneration. Among them, BMP-2 has played an important role because of its ability to regulate the expression of a variety of molecular events contributing to bone formation. ${ }^{\mathbf{9}, 10}$ Furthermore, BMP-2 has received approval from the United States Food and Drug Administration (FDA) to be used in orthopaedic surgery. ${ }^{11}$ Multiple studies demonstrated that BMP-2 can improve the expression of osteogenic markers during osteogenic differentiation in vitro such as osteopontin, osteocalcin, and alkaline phosphatase.,12,13 However, BMP-2 acts mainly on the osteoblastic differentiation and is less effective for cell proliferation, which means that the bone-forming effect of BMP-2 alone is less effective in the case of a large bone defect due to the insufficient number of cells. ${ }^{\mathbf{1 4 , 1 5}}$ To overcome this drawback, a strategy for improving the regenerative potential of BMP-2-based bone implants is using the combinations of BMP-2 with other growth factors, which can enhance bone healing and tissue regeneration. IGF-1, as somatomedin C and somatomedin A, is a mitogenic factor that enhances growth in both adult and embryonic cells as well as inhibits cell apoptosis. ${ }^{16}$ It has been shown to enhance osteoblast growth and proliferation, resulting in enhanced osseointegration at the local site. Furthermore, many studies have demonstrated that IGF-1 can promote osteoblast differentiation due to the activation of the mammalian target of rapamycin (mTOR) through the PI3 K/Akt pathway. ${ }^{17}$ Therefore, IGF-1 can effectively regulate the processes necessary for the proliferation and differentiation of cells, promotes bone matrix formation, and has the ability to reinforce BMP-2. Chen et al. found that the combined use of BMP-2 and IGF-1 had better ability to increase ALP expression of periodontal ligament fibroblasts. ${ }^{18}$ For the above reasons, it has gained interest for us to use BMP-2 and IGF-1 in combination to improve the osteointegration of PLGA/HA scaffold.

The conventional method of the combination of growth factors into polymer scaffolds is that growth factors were incorporated directly into the polymer scaffolds during the scaffold fabrication. ${ }^{19,20}$ However, large amounts of organic solvents are used in the preparation of polymer scaffold, which will damage the bioactivity of growth factor. In recent years, the immobilization method has attracted much attention as a new delivery method. ${ }^{21,22}$ It has been reported that immobilized growth factors could compensate for the lack of conventional methods and be retained in the designated location to maintain the stimulation effect for a long time period. Recently, a musselinspired immobilization strategy for immobilization of growth factors on polymer scaffolds via self-polymerization of dopamine has also been utilized to potentiate the regenerative capacity of cells for bone regeneration in vitro and in vivo. ${ }^{23-25}$ Dopamine is a single molecule consisting of a 3,4-dihydroxy-Lphenelalanine (DOPA)-like catechol group and a lysine-like alkylamine group, which can self-polymerize in an alkaline environment (polydopamine, PDA) and adhere to various substrates, including polymers, metals, and metal oxides. ${ }^{26}$ Numerous studies have found that PDA coating can serve as an intermediate for conjugation of biomolecules onto scaffold surfaces without surface pretreatment. ${ }^{26,27}$ Therefore, it is a simple and effective way to immobilize growth factors onto the polymer scaffold and does not require multistep polymer synthesis. Furthermore, it has been reported that PDA coating can significantly promote the adhesion and proliferation of osteoblasts. ${ }^{28}$ Meanwhile, the capacity of PDA to concentrate $\mathrm{Ca}^{2+}$ using its catechol moieties can improved the nucleation of hydroxyapatite..$^{\mathbf{2 9}, \mathbf{3 0}}$ These features can improve the osteointegration of polymer materials.

Thus, in this study, we developed a method to immobilize BMP-2 and IGF-1 onto PLGA/HA porous scaffolds via the polydopamine coating process. The aim of this study was to improve cell affinity and osteogenesis of PLG/HA porous scaffolds for clinical bone repair. The immobilization efficiency and release kinetics of BMP-2 and IGF-1, and the combined effects of BMP-2 and IGF-1 on cell attachment, cell proliferation, osteogenic differentiation, and the ability to repair bone in vivo was explored to investigate the effectiveness of the surface modification with growth factors.

\section{Materials and methods}

\section{Materials}

The chemicals were provided by Sigma-Aldrich. PLGA (lactide/ glycolide ratio $=75 / 25, M_{\mathrm{n}}=147000$ ) was purchased from Changchun SinoBiomaterials Co., Ltd., and HA powder was purchased from Nanjing Emperor Nano Material Co., Ltd. BMP2 and IGF-1 were purchased from UB Biotech. Co. Ltd. Cetylpyridinium chloride (CPC) was purchased from Aladdin Chemistry Co. Ltd.

\section{Fabrication of PLGA/HA porous scaffold}

The PLGA/HA porous scaffolds were prepared by combining phase inversion and particulate leaching method. Briefly, $0.2 \mathrm{~g}$ of HA was dispersed in $1 \mathrm{~mL}$ of NMP ( $N$-methyl-2-pyrrolidone), and $1.8 \mathrm{~g}$ of PLGA was dissolved in $9 \mathrm{~mL}$ of NMP. Then, the HA suspension was blended with the PLGA solution to achieve an HA content of $10 \mathrm{wt} \%$ in the composites. After magnetic stirring at room temperature for $24 \mathrm{~h},(\mathrm{NaCl})$ particulates of $300-450 \mu \mathrm{m}$ in diameter were added into the PLGA/HA/NMP mixture in an internal mixer at $60 \mathrm{rpm}$ for $5 \mathrm{~min}$. The weight ratio of the salt particulates to PLGA/HA was $6: 1$. The role of salt particles is pore-forming agents. During scaffold preparation, the salt particles can removed from the composites by leaching in distilled water, and form big pores in scaffold. These big pores can provide space for cells to growth and migration in scaffold. The mixture was moulded in a homemade glass cylinder with a removable bottom. The salt particles and NMP were subsequently removed from the composites by immersion in distilled water for 3 days with the water exchanged every $12 \mathrm{~h}$. Finally, the PLGA/HA porous scaffolds were obtained after being vacuumdried for 3 days. Pure PLGA porous scaffolds were also prepared in the same conditions. 
PDA-assisted immobilization of BMP-2 and IGF-1 onto PLGA/ HA porous scaffolds

The PDA-coating process was carried out as previously described. ${ }^{26}$ Briefly, the dried PLGA/HA porous scaffolds were immersed in an aqueous solution of dopamine $\left(2 \mathrm{mg} \mathrm{mL}^{-1}\right.$ in $10 \mathrm{mM}$ Tris $\cdot \mathrm{HCl}, \mathrm{pH}=8.5$ ) with mild shaking for $2 \mathrm{~h}$. Then, the PDA-coated PLGA/HA porous scaffold (PDA-PLGA/HA) were washed with deionized water five times to remove unattached dopamine molecules. Afterwards, BMP-2 (500 ng mL ${ }^{-1}$ in PBS) and IGF-1 (100 ng $\mathrm{mL}^{-1}$ in PBS) were introduced onto PDAPLGA/HA porous scaffolds for another $2 \mathrm{~h}$ with continuous shaking by an oscillator at room temperature. Finally, the porous scaffolds were washed with distilled water three times to remove the unattached growth factors. For cell experiment, the final dimension of the all porous scaffold is $0.3 \mathrm{~cm}$ in width, $0.5 \mathrm{~cm}$ in length. For animals experiment, the final dimension of the all porous scaffold is $0.3 \mathrm{~cm}$ in width, $2 \mathrm{~cm}$ in length. Furthermore, the films (PLGA, PLGA/HA, PDA-PLGA/HA and growth factor-immobilized) were also prepared and treated under similar conditions to the porous scaffold.

\section{Surface characterization}

The surface morphology of the PLGA/HA porous scaffolds with or without polydopamine coating was examined by using a scanning electron microscope (ESEM, XL30 FEG, Philips). Energy-dispersive X-ray spectroscopy (EDX, Philips, XL-30W/ TMP, Japan) was employed to detect the elemental composition of the porous scaffolds. The static water contact angle was used to evaluate the surface wettability of scaffolds using a Kruss GmbH DSA $100 \mathrm{Mk} 2$ goniometer.

\section{Cell culture}

Mouse preosteoblasts (MC3T3-E1) were purchased the from Institute of Biochemistry and Cell Biology, Shanghai Institutes for Biological Sciences, Chinese Academy of Sciences, and cultured in high-glucose DMEM supplemented with $10 \%$ foetal bovine serum, $100 \mathrm{U} \mathrm{mL}^{-1}$ penicillin, and $100 \mathrm{mg} \mathrm{mL}^{-1}$ streptomycin at $37{ }^{\circ} \mathrm{C}$ in a humidified atmosphere containing $5 \%$ $\mathrm{CO}_{2}$. The medium was renewed every 2 days. When they reached $80 \%$ confluence, the cells were harvested for the following assessment.

\section{Cell spreading, attachment, and proliferation assays}

Cell proliferation of MC3T3-E1 in virus porous scaffolds was assayed using the 3-(4,5-dimethyl-2-thiazolyl)-2,5-diphenyl tetrazolium bromide (MTT) method. Briefly, the porous scaffolds were sterilized by immersion in $70 \%$ alcohol for $30 \mathrm{~min}$. After being washed by PBS for three times, they were placed in the 24-well tissue culture plates. Then, $1 \mathrm{~mL}$ of MC3T3-E1 cells $\left(2.5 \times 10^{4} \mathrm{~mL}^{-1}\right)$ was seeded onto porous scaffolds, followed by incubation at $37{ }^{\circ} \mathrm{C}$ and $5 \% \mathrm{CO}_{2}$ for 1,4 , and $7 \mathrm{~d}$, respectively. The culture medium was replaced every two days. At the appropriate time point, $100 \mu \mathrm{L}$ of MTT ( $5 \mathrm{mg} \mathrm{mL}^{-1}$ in PBS) was added into each well, and incubated for $4 \mathrm{~h}$ at $37{ }^{\circ} \mathrm{C}$. Once completed, the medium was removed, and $800 \mu \mathrm{L}$ of acidified isopropanol ( $2 \mathrm{~mL}$ of $0.04 \mathrm{~N}$ hydrochloric acid $(\mathrm{HCl})$ in $100 \mathrm{~mL}$ of isopropanol) was added to each well to solubilize the converted dye. After the incubation period, the absorbance at $540 \mathrm{~nm}$ was read with a microplate reader (Tecan Infinite M200).

To investigate the effect of polydopamine coating and growth factors on cell adhesion and spreading, the morphology of adherent cells cultured on various films (PLGA, PLGA/HA, PDAPLGA/HA and growth factor-immobilized) was evaluated after 1 and $4 \mathrm{~d}$. Briefly, after seeding $2.5 \times 10^{4}$ cells for each sample and incubation for 1 and $4 \mathrm{~d}$, cells were fixed with $4.0 \%$ paraformaldehyde for $30 \mathrm{~min}$ followed by rinsing three times. Afterwards, the actin microfilaments of cells were then stained with phalloidin tetramethylrhodamine $\mathrm{B}$ isothiocyanate (Invitrogen) diluted in BSA/PBS $(1 \% \mathrm{w} / \mathrm{v})$ by incubating the cell/film samples for $30 \mathrm{~min}$ at room temperature. Then, the cell nucleus was stained using 4',6-diamidino-2-phenylindole (DAPI; SigmaAldrich, USA). The morphology of the cells attached and spread on each sample was observed with a fluorescence microscope (TE2000-U, Nikon).

\section{Alkaline phosphate (ALP) activity assay}

To evaluate the early osteogenic differentiation of MC3T3-E1 cells, ALP activity was measured. In brief, after incubation for 7 and 14 days on various films, cellular ALP activity was investigated via a pNPP assay. The medium of each well was carefully removed at the end of the incubation. Then, MC3T3-E1 cells were washed three times with PBS, lysed in RIPA buffer frozen at $-80{ }^{\circ} \mathrm{C}$ for $30 \mathrm{~min}$, and thawed at $37{ }^{\circ} \mathrm{C}$. A $500 \mu \mathrm{L}$ portion of pNPP solution was placed in every well away from light and maintained at $37{ }^{\circ} \mathrm{C}$ for $30 \mathrm{~min}$. The reaction was stopped by adding $500 \mu \mathrm{L}$ of $1 \mathrm{~N} \mathrm{NaOH}$, and the ALP activity was read on a multifunction microplate scanner at $405 \mathrm{~nm}$. Measurements were normalized by the number of cells from BCA protein assay.

\section{Mineralization}

Alizarin red S (ARS) staining was performed to assess the calcium deposition by MC3T3-E1 cells incubated on different porous scaffold materials for 14 and 21 days. After being washed three times with PBS for 3 times, the samples were fixed as described in with $4 \%$ glutaraldehyde solution for $30 \mathrm{~min}$. Then, the samples were washed with acidic PBS ( $\mathrm{pH} 4.2)$ for three times and stained with ARS $(50 \mathrm{mM})$ for $30 \mathrm{~min}$ at $37^{\circ} \mathrm{C}$. Subsequently, the stained cells were rinsed with acidic PBS three times and observed under a light microscope. Calcium quantification was measured using cetylpyridinium chloride (CPC) treatment, and ARS stained films were washed with distilled water and treated with $1 \mathrm{~mL}$ of $10 \%$ CPC solution for $1 \mathrm{~h}$ to desorb the calcium ions. Absorbance at $540 \mathrm{~nm}$ was determined using a multifunctional microplate scanner.

\section{Real-time polymerase chain reaction (RT-PCR) analysis}

MC3T3-E1 cells were incubated on various porous scaffolds for 7 and 14 days. Osteogenic relative gene expressions for runtrelated transcription factor 2 (Runx2) and osteopontin (OPN) were assessed by real-time polymerase chain reaction (RT-PCR). At 
the time point, the cells were digested, and total RNA was isolated. Then the cDNA was synthesized using a PrimeScript ${ }^{\mathrm{TM}} \mathrm{RT}$ reagent kit (Takara Bio, Japan) according to the manufacturer's instructions. The expression of osteogenic markers was quantified by qPCR SYBR Green Mix Kit (TaKaRa). Gene-specific primers including glyceraldehyde-3-phosphate dehydrogenase (GAPDH), anti-runt-related transcription factor 2 (Runx2) and osteopontin (OPN) were designed using the primer design software of beacon 5.0 (Table 1). qRT-PCR was conducted by StepOnePlus Real-Time PCR System (Applied Biosystems, CA) and expression levels were obtained using threshold cycles $(\mathrm{Ct})$ that were determined by the iCycler iQ Detection System software. For quantitative realtime PCR, $10 \mu \mathrm{L}$ of SYBR Premix Ex Taq ${ }^{\mathrm{TM}}, 6.8 \mu \mathrm{L}$ of $\mathrm{dH}_{2} \mathrm{O}, 0.4 \mu \mathrm{L}$ of each forward and reverse primer, $0.4 \mu \mathrm{L}$ of Rox, and $2.0 \mu \mathrm{L}$ of cDNA template were used in a final reaction volume of $20 \mu \mathrm{L}$. The PCR amplification cycles included denaturation for $5 \mathrm{~s}$ at $95^{\circ} \mathrm{C}$, annealing, and extension for $34 \mathrm{~s}$ at $56{ }^{\circ} \mathrm{C}$ for 40 cycles. Data collection was enabled at $56{ }^{\circ} \mathrm{C}$ in each cycle. The relative gene expression levels of Runx 2 and OPN were normalized to GAPDH. All experiments were done in triplicate to obtain the average data.

\section{Repair for rabbit radius defects}

The PLGA, PLGA/HA, PDA-PLGA/HA and growth factorsimmobilized porous scaffolds were implanted into rabbit radius defects. Bilateral critically sized defects of New Zealand white rabbits with body weights of $2.0-2.5 \mathrm{~kg}$ were created in the radius by removing $20 \mathrm{~mm}$ of midshaft diaphyseal bone. The wounds were closed with silk threads in layers. After surgery, the rabbits were returned to their cages and allowed to move freely. All rabbits were injected daily with penicillin intramuscularly at a dose of 200000 units for each one for three days. In the untreated group, nothing was implanted in the defect for the control. Three replicates were set for each group. Animals were purchased and kept in the Institute of Experimental Animal of Jilin University. All animal procedures were performed in accordance with the Guidelines for Care and Use of Laboratory Animals of Jilin University and approved by the Animal Ethics Committee of Jilin University.

\section{X-ray examination}

In vivo osteogenesis at the rabbit radius defects repaired with different porous scaffolds were examined with Digital Radiograph (DR, KODAK CR 400 plus Filmless Radiology System, USA) at 0,4 , and 8 weeks after surgery to follow the healing

Table 1 List of genes and primer nucleotide sequences

\begin{tabular}{ll}
\hline Gene & Primer sequence $\left(5^{\prime}-3^{\prime}\right)$ \\
\hline OPN & F: TCAGGACAACAACGGAAAGGG \\
& R: GGAACTTGCTTGACTATCGATCAC \\
Runx2 & F: GCCCTCATCCTTCACTCCAAG \\
& R: GGTCAGTCAGTGCCTTCCTC \\
GAPDH & F: AATGTGTCCGTCGTGGATCTG \\
& R: CAACCTGGTCCTCAGTGTAGC
\end{tabular}

process at the resection sites, and points were allotted according to the Lane-Sandhu scoring system. The rabbits were exposed to X-ray in a prone position during anaesthetization. Afterwards, X-ray films were exported as TIF images. All the points were given by five independent examiners who were trained in the Lane-Sandhu system. The points were identified according to the degree of bone formation, connections, and bone marrow recanalization. All X-rays films were scored by the Lane-Sandhu Radiographic Scoring System. ${ }^{31}$

\section{Statistical analysis}

The data were analysed using Origin 8.0 software. All quantitative data were presented as the mean \pm standard deviation. Statistical comparisons were carried out using one-way analysis of variance (ANOVA). A $p$-value of $<0.05$ was considered to be statistically significant.

\section{Results and discussion}

\section{Surface characterization of porous scaffolds}

The morphology and microstructure of the PLGA, PLGA/HA and PDA-PLGA/HA porous scaffolds were observed by SEM. As shown in Fig. 1A, the pores of all porous scaffolds were interconnected and irregular, and the surface of pores had a honeycomb-like structure composed of microvoids with diameters of 2-4 $\mu \mathrm{m}$, which was suitable for cell infiltration and the transportation of oxygen and nutrients. Compared to the PLGA scaffold, the pore wall surfaces of the PLGA/HA scaffolds were rougher than those of PLGA scaffolds because of the incorporation of HA crystals. After PDA coating, the PDA-PLGA/HA porous scaffold displayed similar surface morphology to the PLGA/HA scaffold, but was covered with several polydopamine
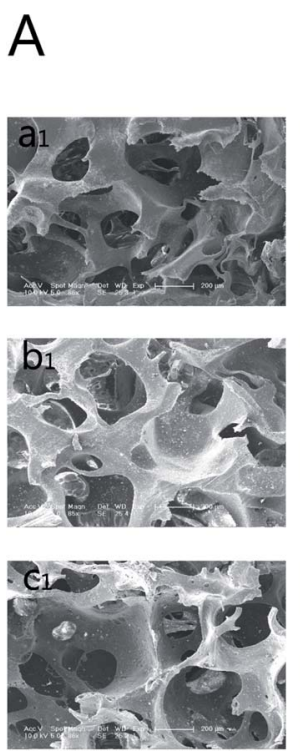
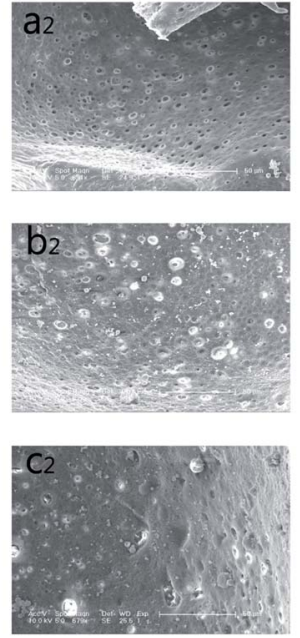

B

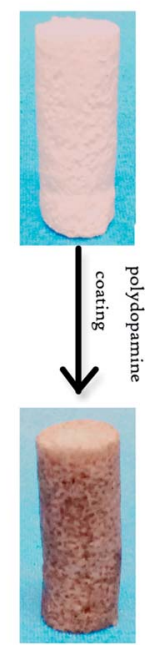

Fig. 1 (A) SEM images of PLGA (a1 and a2), PLGA/HA (b1 and b2) and PDA-PLGA/HA (c1 and c2) porous scaffolds. Bar lengths are $200 \mu \mathrm{m}$ $(a-1, b-1$ and $c-1)$ and $50 \mu \mathrm{m}(a-2, b-2$, and $c-2)$. (B) The macroscopic view of untreated porous scaffolds and PDA-coated porous scaffolds. 
precipitate particles, and the surface roughness of the porous scaffold increased. Furthermore, the PDA surface coating can be easily distinguished by the colour change of the porous scaffolds (Fig. 1B). The interactions between the PDA coating and the PLGA/HA substrate include covalent and noncovalent interactions such as the hydrogen bonding interaction, $\pi-\pi$ interaction, and electrostatic interaction. Furthermore, the coexistence of catechol and amine (lysine) groups is a crucial factor for achieving adhesion to a wide spectrum of materials, and dopamine as a small-molecule compound that contains both functional groups. The porous scaffolds were also analysed with EDX. As shown in Fig. 2, it was found that the major elements in the pure PLGA porous scaffolds were carbon (C) and oxygen (O). The EDX element analysis showed that there was existence of phosphorus (P) and calcium (Ca) on the surface of the PLGA/HA porous scaffolds compared to that of PLGA scaffolds. After polydopamine coating, nitrogen (N) was found in the EDX spectra of PDA-PLGA/HA, whereas $\mathrm{N}$ was not found in the EDX spectra of PLGA and PLGA/HA scaffolds. The results of the SEM and EDX spectra suggested that PDA was successfully coated onto the surfaces of PLGA/HA porous scaffolds.

\section{Contact angle measurement}

The hydrophilicity of the materials plays an important role in interacting with cells. In addition, hydrophilic surfaces are better for protein adsorption, such as fibronectin, vitronectin and various growth factors than hydrophobic surfaces, which can not only reduce the amount of growth factors, but also make the biomaterials retain long-term bioactivity. The contact angles of different porous scaffolds were obtained by calculating the included angle between the water droplet and

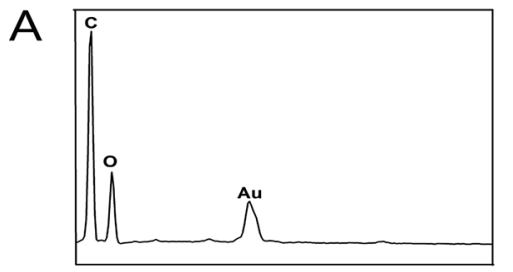

B
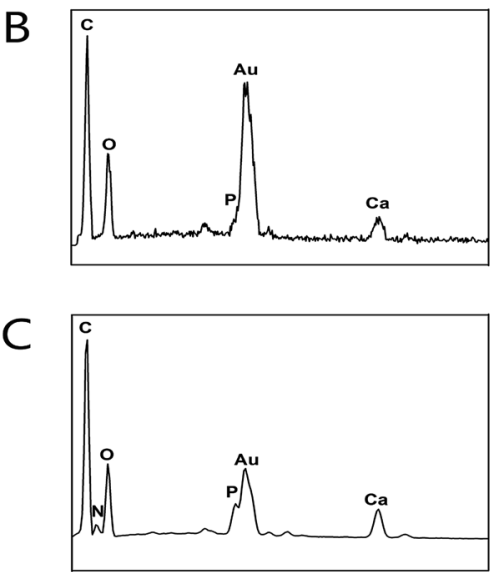

Fig. 2 EDX analysis of PLGA (A), PLGA/HA (B) and PDA-PLGA/HA (C) porous scaffolds. horizontal plane, as shown in Fig. 3. Compared with pristine PLGA, the PLGA/HA samples showed decreased contact angles from $95.6^{\circ} \pm 6.2^{\circ}$ to $84.5^{\circ} \pm 7.5^{\circ}$ due to the exposure of HA on the surface. For PDA-PLGA/HA samples, the wettability was further improved by PDA coating, as evidenced by a dramatic decrease in the water contact angle to $48.4^{\circ} \pm 5.4^{\circ}$. Furthermore, the contact angle was further decreased for BMP-2 and IGF-1-immobilized PDA-PLGA/HA scaffolds, which indicated that the wettability of PDA-PLGA/HA porous scaffolds was improved by the adsorbed growth factors to some extent. The results of contact angle measurement indicated that the hydrophilicity of porous scaffolds was improved by PDA coating and PDA-mediated growth factors immobilization. Since the optimum range of water contact angles for cell culture substrates is between $5^{\circ}$ and $40^{\circ}$, and the proliferation rate of cells can be improved if they grow on the materials with such a water contact angle. ${ }^{32,33}$ The above results show that BMP-2 and IGF-1-immobilized PDA-PLGA/HA porous scaffolds can provide an appropriate microenvironment for promoting the attachment and proliferation of cells.

\section{Adsorption and release of IGF-1 and BMP-2}

The binding efficiency and release profiles of IGF-1 and BMP-2 on PLGA/HA and PDA-PLGA/HA scaffolds were identified by ELISA. As shown in Fig. 4(A and B), it could be seen that the IGF1 and BMP-2 binding efficiency of PDA-PLGA/HA scaffolds was higher than that of the PLGA/HA scaffolds. The efficiency of IGF1 and BMP-2 immobilization on PDA-PLGA/HA scaffolds was approximately 2.5 and 2.7 times more than that on un-coated scaffolds, respectively. This result demonstrated that the polydopamine coating on the surface of porous scaffold could
A

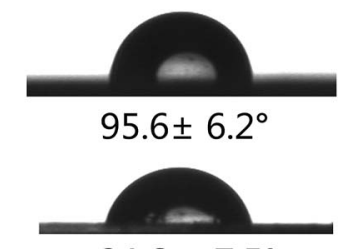

$84.6 \pm 7.5^{\circ}$

C

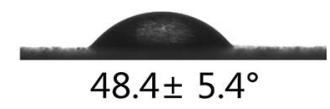

D

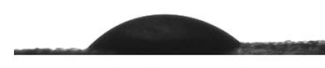

$45.9 \pm 9.1^{\circ}$

E

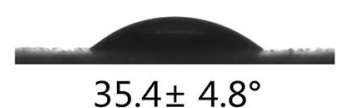

$\mathrm{F}$

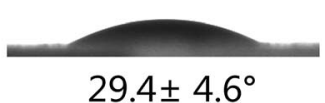

Fig. 3 Water contact angles measurement for different porous scaffolds (A) PLGA, (B) PLGA/HA, (C) PDA-PLGA/HA, (D) PDA-PLGA/HA/ IGF-1, (E) PDA-PLGA/HA/BMP-2, (F) PDA-PLGA/HA/IGF-1/BMP-2. 

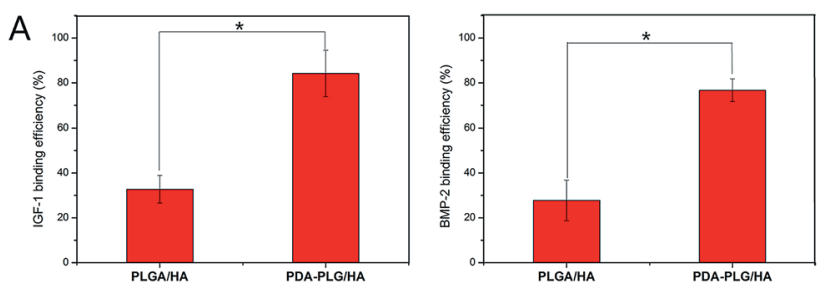

B
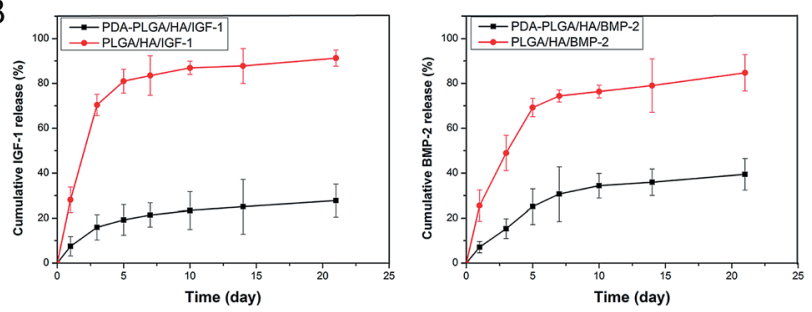

Fig. 4 Binding efficiency of IGF-1 and BMP-2 to PLGA/HA and PDAPLGA/HA porous scaffolds (A). Cumulative release of IGF-1 and BMP-2 from PLGA/HA and PDA-PLGA/HA porous scaffolds (B). $P<0.05$, error bars represent standard deviation for $n=3$.

effectively increase growth factors binding efficiency of the scaffold. Furthermore, investigation of the BMP-2 and IGF-1 release profile showed that there was a burst release with $19 \%$ (IGF-1) and 25\% (BMP-2) of the total growth factors released from the PDA-PLGA/HA porous scaffolds within five days; the release subsequently slowed down with approximately 27\% (IGF-1) and 39\% (BMP-2) of the total growth factors released after $21 \mathrm{~d}$, respectively. The released IGF-1 and BMP-2 exhibited similar release behaviours. However, for the PLGA/HA porous scaffolds, a burst release with $81 \%$ and $69 \%$ of IGF-1 and BMP-2 released from the PLGA/HA scaffold within five days was found; at $21 \mathrm{~d}, 91 \%$ IGF-1 and $84 \%$ BMP-2 was already released from the PLGA/HA scaffold. The above results indicated that the PDA coating could effectively immobilize growth factors on the surface of the scaffold and effectively reduce the burst release of growth factor, and immobilized growth factor keep a sustained release rate compared to physical absorption coating, which can not only reduce the amount of growth factors, but also make the composites perform long-term osteoconductivity. Previous studies have verified that various biomolecules can easily conjugate onto the surface of the PDA-coated materials, depending on the reaction of catechols with thiols and amines, such as BMP-2, VEGF and bFGF. ${ }^{26,27,34}$ Zhou et al. successfully immobilized P24 peptide on polydopamine coated nHA/RHLC/PLA scaffolds and found that PDA-assisted coating could effectively immobilize P24 peptide on the surface of nHA/RHLC/PLA scaffold and allowed for its controlled release. ${ }^{35}$ Another study found that immobilized bFGF on PDA coated PLGA electrospun fibrous scaffolds showed higher bFGF immobilization efficiency than PLGA electrospun fibrous scaffolds. ${ }^{26}$ In this study, our results are consistent with the results of those studies, indicating that polydopamine coating is an efficient and simple method for conjugation of bioactive factors.

\section{Cell adhesion and proliferation}

The biocompatibility of the PDA-PLGA/HA scaffold loaded with IGF-1 or/and BMP-2 was evaluated via a cell adhesion and proliferation assay. MC3T3-E1 cells were seeded and cultured on different porous scaffolds (PLGA, PLGA/HA, PDA-PLGA/HA, and PDA-PLGA/HA loaded with IGF-1 or/and BMP-2), and the cell proliferation in different porous scaffolds was assessed on days 1, 4 and 7 with the MTT assay. As shown in Fig. 5, the cells, seeded on the untreated PLGA porous scaffolds, showed the lowest levels of cell viability at each time points. Compared to PLGA porous scaffolds, cells exhibited a better proliferation ability on the PLGA/HA porous scaffolds at 4 and 7 days. The rough surface could have a positive impact on the adherence of the cells. Previous study has demonstrated that $10 \%$ HA could promote cell adhesion on the PLGA, due to its rough surface after HA doping. ${ }^{36}$ After polydopamine coating, the cell proliferation was significantly greater in the PD-PLGA/HA scaffold compared to PLGA and PLGA/HA at different time points ( $p<$ 0.05). Many studies have shown that the amine and hydroxyl

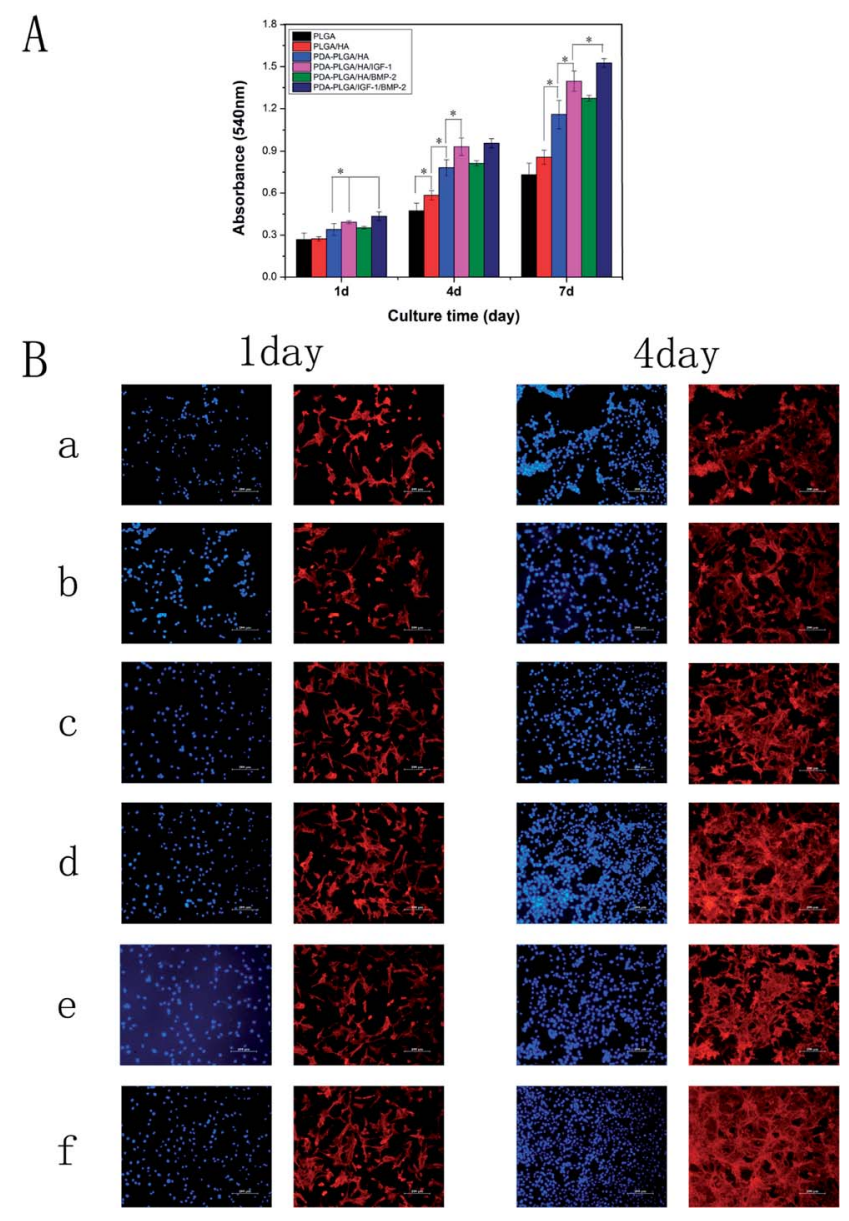

Fig. 5 (A) Proliferation of MC3T3-E1 cells in different porous scaffolds by MTT $(p<0.05, n=4)$. (B) Fluorescent staining observation of the MC3T3-E1 cells cultured on different composites (a: PLGA, b: PLGA/ HA, c: PDA-PLGA/HA, d: PDA-PLGA/HA/IGF-1, e: PDA-PLGA/HA/ BMP-2, f: PDA-PLGA/HA/IGF-1/BMP-2) for 1 and 4 days. All scale bar lengths are $200 \mu \mathrm{m}$. 
groups on the surface of the scaffold benefit cell adhesion, spread, and growth. ${ }^{37,38}$ PDA coating can produced a large amount of amine and hydroxyl groups and exhibiting a special adhesion behavior, which could promote the adhesion and proliferation of cells. When the MC3T3-E1 cells were cultured on the PDA-PLGA/HA scaffold loaded with IGF-1 or/and BMP-2, a higher OD value was found on the growth factor-immobilized scaffold compared with the pristine PDA-PLGA/HA scaffold, and the proliferation and viability of cells increased with the addition of IGF-1. However, for the PDA-PLGA/HA/BMP-2 porous scaffold, the proliferation and viability of cells were significantly lower than those on the PDA-PLGA/HA/IGF-1 and PDA-PLGA/ HA/IGF-1/BMP-2 porous scaffolds, suggesting that IGF-1 played a more important role in MC3T3-E1 cell proliferation. Many studies have reported that IGF-1 can significantly promote cell proliferation as a therapeutic factor either alone or in combination with other active ingredients. ${ }^{17,18}$ Among the various porous scaffolds, the PDA-PLGA/HA/IGF-1/BMP-2 scaffold was most effective at enhancing cell proliferation in this study, indicating that the combination of IGF-1 and BMP-2 had a synergistic effect in terms of cell proliferation.

It is well known that cell adhesion is an important cellular behavior because it directly affects proliferation. Therefore, we also analysed the effect of the HA, polydopamine coating and immobilized IGF-1 or/and BMP-2 on the adhesion of MC3T3-E1 cells. As shown in Fig. 5B, MC3T3-E1 cells were fluorescently stained to investigate cell adhesion and migration behavior. After $1 \mathrm{~d}$ of culture, cells on the PDA-PLGA/HA substrates exhibited a greater spread with a better cytoskeleton than those on PLGA and PLGA/HA substrates. After IGF-1 was immobilized, the cell adhesion on the PDA-PLGA/HA/IGF-1 and PDA-PLGA/ HA/IGF-1/BMP-2 composites was better than that on the PDAPLGA/HA substrates, indicating that IGF-1 is beneficial for cell growth and cell-cell communication. Meanwhile, it was also found that the immobilization of BMP-2 had little effect on the adhesion of MC3T3-E1 cells. After $4 \mathrm{~d}$ of culture, it was found that the cell spreading areas on PDA-PLGA/HA were slightly wider than those on PLGA and PLGA/HA. Furthermore, the density of cells on IGF-1 or/and BMP-2 immobilization substrates was higher than the other groups and covered the films. However, compared to PDA-PLGA/IGF-1 and PDA-PLGA/ HA/IGF-1/BMP-2, the cell adhesion was unsatisfactory with only BMP-2 adsorbed onto the PDA-PLG/HA substrates. These results of cell adhesion are consistent with the MTT analysis, and further supported that IGF-1 and BMP-2 dual immobilization on PDA-PLGA/HA porous scaffolds can better promote MC3T3-E1 cell adhesion and proliferation.

\section{Alkaline phosphatase activity}

ALP plays a crucial role in the initiation of the mineralization process by cell differentiation, which peaked during the preosteoblast stage of differentiation..$^{39}$ Therefore, the osteogenic differentiation of MC3T3-E1 cells cultured on different porous scaffolds was assessed by measuring ALP activity. As shown in Fig. 6, the ALP activity of MC3T3-E1 cells cultured on PLGA/HA scaffolds was significantly higher than that on PLGA scaffolds ( $p$
A
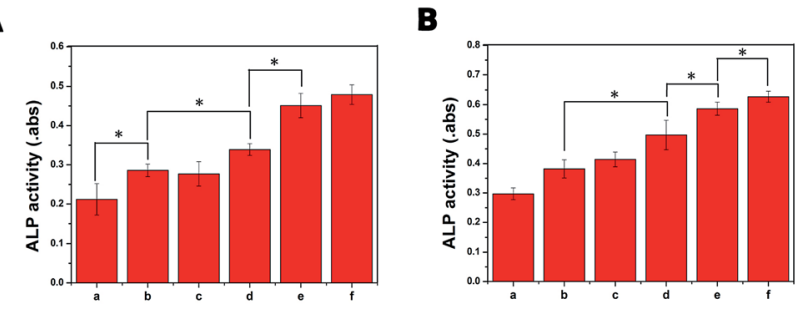

Fig. 6 ALP activity of MC3T3-E1 cells on different porous scaffolds at 7 (A) and 14 (B) days: (a) PLGA; (b) PLGA/HA; (c) PDA-PLGA/HA; (d) PDAPLGA/HA/IGF-1; (e) PDA-PLGA/HA/BMP-2; and (f) PDA-PLGA/HA/ IGF-1/BMP-2. $P<0.05, n=4$.

$<0.05)$. As the primary mineral component in mature bone, previous studies found that $10 \%$ HA could induce cell osteogenic differentiation and increase the ALP activity of cells. ${ }^{40}$ After polydopamine coating, there was no significant differences between PLGA/HA and PDA-PLGA/HA porous scaffolds, suggesting that PDA coating played little role on cell early osteogenesis differentiation. A great increase in ALP activity in cells was found on the BMP-2-or IGF-1/BMP-2-immobilized porous scaffolds, higher than those of cells on the other porous scaffolds at both 7 and 14 days $(p<0.05)$, indicating that the osteodifferentiation ability was stronger for the BMP-2immobilized scaffolds than others. Furthermore, the ALP activity of MC3T3-E1 cells on IGF-1-immobilized porous scaffolds was better than that of PDA-PLGA/HA and PLGA/HA porous scaffolds. It was reported that IGF-1 could significantly increase ALP expression of cells..$^{41}$ More importantly, we found that the combination of BMP-2 and IGF-1 exhibited higher ALP activity than BMP-2 alone, suggesting that dual release of BMP-2 and IGF-I could better enhance osteogenic differentiation of cells.

\section{Cell mineralization}

One of the pieces of evidences for differentiation of cells to the osteogenic lineage is their capability to mineralize inorganic phosphates at the middle of the differentiation period. The levels of calcium deposition at different composites were evaluated by ARS staining, which can bind to $\mathrm{Ca}^{2+}$ in mineralized ECM showing bright red stains. As shown in Fig. 7, after 21 days of culture, the alizarin red stain showed slight reddish dots on PLGA/HA substrates, but almost no positive stains were found on pure PLGA substrates, indicating that HA could induce cell osteogenic differentiation. After polydopamine coating, the red calcium nodules in MC3T3-E1 cells on PDA-PLGA/HA substrates were increased compared with the cells growing on PLGA/HA substrates. We speculate that the capacity of polydopamine coating to concentrate $\mathrm{Ca}^{2+}$ could improve the nucleation of $\mathrm{HA}$, which could result in enhanced cell osteogenesis mineralization. After IGF-1 immobilization, there was no significant differences between PDA-PLGA/HA and PDA-PLGA/HA/IGF-1 substrates. However, the substrates incorporating BMP-2 or IGF-1/BMP-2 exhibited the largest and most organized nodules, suggesting that the immobilized BMP-2 possesses better osteoinductive ability, which is stronger than IGF-1. Several 

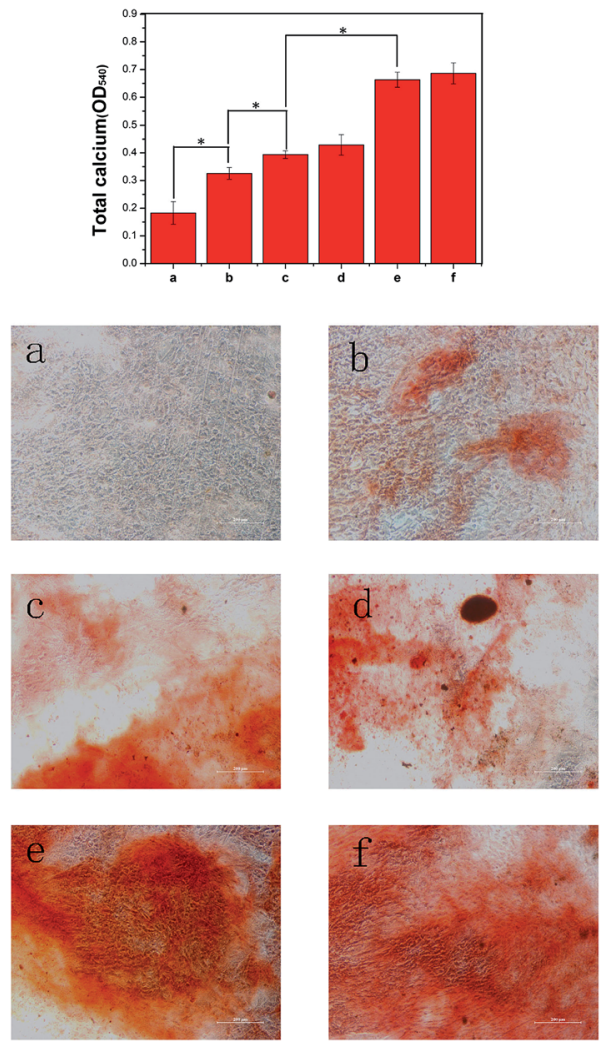

Fig. 7 Alizarin Red staining of MC3T3-E1 cells cultured on PLGA (a), PLGA/HA (b), PDA-PLGA/HA (c), PDA-PLGA/HA/IGF-1 (d), PDA-PLGA/ HA/BMP-2 (e) and PDA-PLGA/HA/IGF-1/BMP-2 (f) films at $21 \mathrm{~d}$. All scale bar lengths are $200 \mu \mathrm{m} . P<0.05, n=4$.

studies have shown that the excellent bone induction activity of BMP-2 was largely due to the mineralization effect of BMP-2 on cell. ${ }^{10,42}$

The assessment of quantitative cell mineralization was performed by extracting ARS with 10\% CPC. After 21 days of culture, the total calcium content in deposited minerals on PDPLGA/HA substrates was significantly higher than that on PLGA/ HA and PLGA substrates. In accordance with the ALP results, the highest calcium content in deposited minerals was still observed with the BMP-2 or BMP-2/IGF-1-immobilized PDAPLGA/HA substrates, indicating that the incorporation of IGF1 and BMP-2 via PDA coating on PLGA/HA substrates significantly facilitates the calcium mineralization of MC3T3-E1 cells.

\section{Quantitative real-time polymerase chain reaction}

There is a certain up-regulation or down-regulation of genes associated with cell proliferation, ECM maturation and ECM mineralization. For example, Runx 2 is an early differentiation marker observed at the early stage of differentiation, and OPN expression is observed at the middle/late stage. In this study, the expression of osteogenic markers (Runx2 and OPN) of MC3T3-E1 cells after $7 \mathrm{~d}$ of culture on different porous scaffolds was quantitatively analysed using qRT-PCR and is shown in Fig. 8. In this study, up-regulation of Runx 2 and OPN gene expression was found in PLGA/HA samples, conforming to the
A

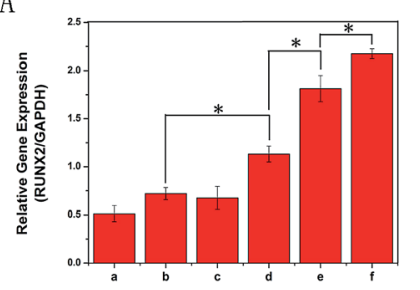

B

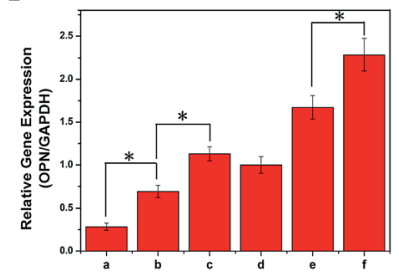

Fig. 8 Quantitative real-time PCR analysis of (A) Runx2 and (B) OPN after culturing MC-3T3-E1 cells for 7 days. (a) PLGA; (b) PLGA/HA; (c) PDA-PLGA/HA; (d) PDA-PLGA/HA/IGF-1; (e) PDA-PLGA/HA/BMP-2; (f) PDA-PLGA/HA/IGF-1/BMP-2. $P<0.05, n=3$.

osteoinductivity of HA. After polydopamine coating, it was found that only the expression of OPN was increased significantly for PDA coating alone on PLGA/HA porous scaffolds without growth factors immobilization. After IGF-1 or/and BMP2 immobilization, the expression levels of Runx2 and OPN in the PDA-PLGA/HA/IGF-1/BMP-2 samples were obviously higher than those in PDA-PLGA/HA/IGF-1 and PDA-PLGA/HA/BMP-2 alone at 7 days. This result further demonstrated that the combination of IGF-1 and BMP-2 had a synergistic effect in terms of osteogenic induction. Furthermore, compared to pristine PDA-PLGA/HA porous scaffolds, the levels of Runx2 gene expression from the PDA-PLGA/HA/IGF-1 groups were higher, but there was no significant increase in OPN expression. In this study, we found that IGF-1 could, but slightly, promote the osteogenic differentiation of cells. However, when combined with BMP-2, IGF-1 showed additive effects on osteogenesis. The results of bone-related genes expression showed the same trends as observed from ALP activity and cell mineralization, indicating that the modification with BMP-2 and IGF1 via PDA coating on the PLGA/HA porous scaffolds can effectively enhance osteodifferentiation of MC3T3-E1 cells.

\section{Bone healing}

The radiographs were taken at post surgery 4 and 8 weeks to detect repair of the rabbit radius defects. The radiographs of the rabbit radius defects implanted with porous scaffolds of PLGA, PLGA/HA, PDA-PLGA/HA and growth factor-immobilized porous scaffolds are shown in Fig. 9. At 4 weeks post-surgery, distinct bone calluses emerged at the defect areas in the PLGA/HA, PDA-PLGA/HA and growth factors-immobilized porous scaffold groups. However, there was only a small quantity of bone callus at the ends of defect areas near to the neighbouring bone in the blank and PLGA groups. There was a small gap between new bone in the PLGA/HA and PDA-PLGA/ HA group, and the area of new bone in the group of PDA-PLGA/ HA was larger than that of PLGA/HA. Among all porous scaffolds, the PDA-PLGA/HA/BMP-2 and PDA-PLGA/HA/IGF-1/BMP2 group had the largest area of newly formed bone. Meanwhile, at 8 weeks after implantation, in the blank and PLGA group, only a small amount of newly formed bone were observed along the defect margins, indicating that pure PLGA and blank groups had only limited ability to induce bone callus formation at the ends of bone defects and failed to unite. In PLGA/HA group, 


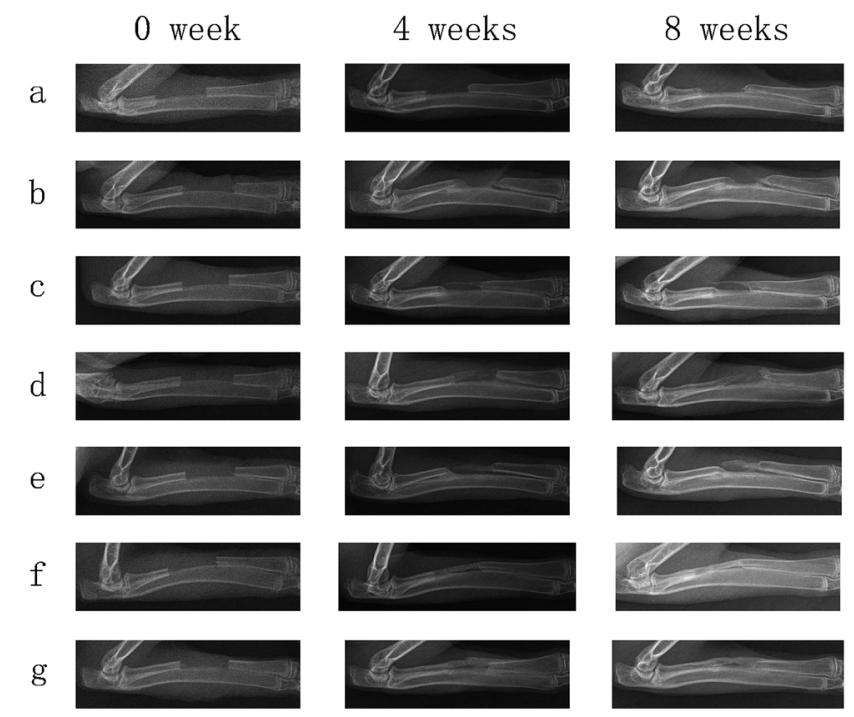

Fig. 9 Digital radiographs of rabbit radius defects of (a) blank and implanted with (b) PLGA, (c) PLGA/HA, (d) PA-PLGA/HA, (e) PDAPLGA/HA/IGF-1, (f) PDA-PLGA/HA/BMP-2 and (g) PDA-PLGA/HA/IGF1/BMP-2 porous scaffolds at 0 weeks, 4 weeks, and 8 weeks.

uniform radiopaque masses were scattered throughout the defect site, but part of the defect was still not filled with calcified tissues. The PDA-PLGA/HA, and PDA-PLGA/HA/IGF-1 groups showed a higher calcified callus than PLGA/HA group. Compared to the other groups, the area of the newly formed bone was more, and the density was higher in the PDA-PLGA/ HA/BMP-2 and PDA-PLGA/HA/IGF-1/BMP-2 groups, indicating that BMP-2-immobilized porous scaffolds exhibited the highest osteoconductivity and the best bone healing quality. As shown in Fig. 10, Lane-Sandhu scoring was performed on the bone defect sites of all the groups. The scores obtained from the blank and PLGA groups were lower than those from other groups. The average scores obtained from the PDA-PLGA/HA and PDA-PLGA/HA/IGF-1 groups were higher than that from PLGA/HA group. Consistent with the results of ALP activity, cell

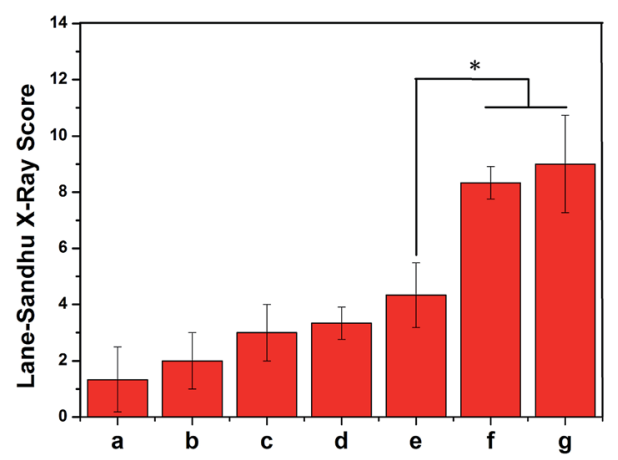

Fig. 10 Lane-Sandhu X-ray scores in (a) blank and implanted with (b) PLGA, (c) PLGA/HA, (d) PA-PLGA/HA, (e) PDA-PLGA/HA/IGF-1, (f) PDAPLGA/HA/BMP-2 and (g) PDA-PLGA/HA/IGF-1/BMP-2 groups at 8 weeks post-surgery. Data are presented as mean $\pm \mathrm{SD}(n=3, * p<$ 0.05). mineralization and osteogenesis-related gene expression, the Lane-Sandhu X-ray scores of all groups revealed that the PDAPLGA/HA/IGF-1/BMP-2 group had the highest score. The results confirmed a significantly greater healing percentage in the PDA-PLGA/HA/IGF-1/BMP-2 group compared with the other groups at each time point.

Bone defects, caused by physical trauma, deformity, degeneration and cancer, have produced high demand for bone grafts. In recent years, with the development of biological materials science, bone tissue engineering shows great potential in developing novel biomaterials as bone grafts for bone regeneration therapies. It is well-recognized that an ideal bone graft should possess both osteoconductivity and osteoinductivity for favourable cell attachment, proliferation and differentiation. To fabricate ideal bone grafts, one strategy is that modification of biomaterials with growth factors or other bioactive molecules, which can effectively improve the bioactivity of bone grafts. At present, various growth factors, such as BMP-2, IGF-1 and bFGF, are employed to induce osteogenic differentiation and promote bone regeneration. ${ }^{13,22,42}$ However, the abilities of single growth factors are limited. For example, BMP-2 is a proven strong osteoinductive factor that stimulates cell osteogenesis differentiation, but has little effect on cell adhesion and proliferation. Therefore, it has gained great attention to improve the differentiation/proliferation potential of bone grafts through the combination or sequential use of growth factors. It was reported that the combined treatment of BMP-2 and bFGF had a better ability to promote bone regeneration than the single use of either BMP-2 or bFGF. ${ }^{43}$ Liu et al. found that IGF-I enhanced alkaline phosphatase (ALP) activity in BMP-7-treated adipose-derived mesenchymal stem cells. ${ }^{44}$ On the other hand, the poor hydrophilicity and lack of functional groups of polymers materials often result in lower growth factor loading efficiency. Therefore, various delivery systems of growth factors have been studied. In this study, BMP-2 and IGF-1 were successfully immobilized on the PLGA/HA porous scaffolds via the PDA-coating method. Previous studies have demonstrated that polydopamine coating can effectively immobilize and deliver growth factors and other biomolecules..$^{26,27,35}$ Zhao et al. used PDA coatings to immobilize BMP-2 on PLGA/HA fibrous scaffold, and they found that the PDA coatings continuously released BMP-2 in a sustained manner for at least 20 days. $^{\mathbf{4 5}}$ Furthermore, it has been shown that PDA coating could promote the adhesion and proliferation of cells on various surfaces. These results are consistent with our findings that PDA coating can effectively immobilize BMP-2 and IGF-1, and sustain the release kinetics compared to un-coated scaffolds. Through in vitro and vivo experiments, the IGF-1 and BMP-2immobilized PDA-PDA/HA porous scaffolds could efficiently promote adhesion, proliferation and osteogenic differentiation of MC3T3-E1 cells in vitro and repair rabbit radius defects in vivo. The combined use of IGF-1 and BMP-2 in the PDA-PLGA/ HA porous scaffolds preserved the osteogenic nature of BMP-2 without diminishing the cell proliferative effect of IGF-1. More importantly, IGF-1 and BMP-2 showed an additive effect in promoting the proliferation and osteogenic differentiation of MC3T3-E1 cells. Thus, we can speculate that the IGF-1 and BMP- 
2-immobilized PDA-PDA/HA porous scaffold may be one of the bioactive polymeric scaffolds for bone defect repair. In future work, we will further investigate the biological properties of the growth factor-modified porous scaffolds to obtain more functional porous scaffolds.

\section{Conclusion}

In this study, PLGA/HA porous scaffold was successfully modified through PDA-assisted immobilization of IGF-1 and BMP-2 using a mussel-inspired biomimetic coating process. The polydopamine coating on the PLGA/HA porous scaffolds can increase the binding sites of porous scaffold to growth factors, which can effectively enhance BMP-2 and IGF-1 binding on surface of the PLGA/HA scaffold, and the IGF-1 and BMP-2 bound on the PLGA/HA porous scaffolds can be slowly released over three weeks in vitro. In vitro and vivo studies including MTT assay, ALP activity, calcium deposition, qRTPCR analysis, and radius bone defect repair indicated that the IGF-1 and BMP-2 immobilized PDA-PDA/HA porous scaffold possess excellent bioactivities for supporting the adhesion, proliferation, and osteogenic differentiation of cell and rabbit radius defect repair. These findings indicated that PDA-assisted surface modification could be an effective, simple method to immobilize growth factors onto biomaterial surfaces, and the IGF-1 and BMP-2 immobilized PDA-PDA/HA porous scaffold are promising candidate materials for bone tissue engineering.

\section{Conflicts of interest}

The authors declare no competing financial interest.

\section{Acknowledgements}

This study was financially supported by the grants from the National Natural Science Foundation of China (No. 81672263, No. 31572217), Jilin Innovation Fund for Small and Mediumsized Technology-based (SC201502001), Jilin Scientific and Technological Development Program (20160101027JC), and the Graduate Innovation Fund of Jilin University (2017031).

\section{References}

1 H. Shen, X. X. Hu, F. Yang, J. Z. Bei and S. G. Wang, Acta Biomater., 2010, 6, 455-465.

2 S. S. Kim, M. S. Park, O. Jeon, C. Y. Choi and B. S. Kim, Biomaterials, 2006, 27, 1399-1409.

3 Z. Heydari, D. Mohebbi-Kalhori and M. S. Afarani, Mater. Sci. Eng., C, 2017, 81, 127-132.

4 M. Karpov, M. Laczka, P. S. Leboy and A. M. Osyczka, J. Biomed. Mater. Res., Part A, 2008, 84, 718-726.

5 X. X. Hu, H. Shen, F. Yang, X. J. Liang, S. G. Wang and D. C. Wu, Appl. Surf. Sci., 2014, 292, 764-772.

6 S. Lee, K. M. Kim and Y. K. Lee, Tissue Eng. Regener. Med., 2010, 7, 561-565.

7 W. Shao, J. He, F. Sang, Q. Wang, L. Chen, S. Cui and B. Ding, Mater. Sci. Eng., C, 2016, 62, 823-834.
8 L. Liao, S. Yang, R. J. Miron, J. C. Wei, Y. F. Zhang and M. Zhang, PLoS One, 2014, 9, e105876.

9 S. Park, H. A. Heo, K. B. Lee, H. G. Kim and S. W. Pyo, Implant Dentistry, 2017, 26, 4-11.

10 C. A. Luppen, E. Smith, L. Spevak, A. L. Boskey and B. Frenkel, J. Bone Miner. Res., 2003, 18, 1186-1197.

11 X. F. Shen, Y. X. Zhang, Y. Gu, Y. Xu, Y. Liu, B. Li and L. Chen, Biomaterials, 2016, 106, 205-216.

12 Y. Takuwa, C. Ohse, E. A. Wang, J. M. Wozney and K. Yamashita, Biochem. Biophys. Res. Commun., 1991, 174, 96-101.

13 W. G. La, M. J. Jung, J. K. Yoon, S. H. Bhang, H. K. Jang, T. J. Lee, H. H. Yoon, J. Y. Shin and B. S. Kim, Carbon, 2014, 78, 428-438.

14 M. Hughes-Fulford and C. F. Li, J. Orthop. Surg. Res., 2011, DOI: $10.1186 / 1749-799 X-6-8$.

15 A. Yamaguchi, T. Katagiri, T. Ikeda, J. M. Wozney, V. Rosen, E. A. Wang, A. J. Kahn, T. Suda and S. Yoshiki, J. Cell Biol., 1991, 113, 681-687.

16 A. Jaklenec, A. Hinckfuss, B. Bilgen, D. M. Ciombor, R. Aaron and E. Mathiowitz, Biomaterials, 2008, 29, 1518-1525.

17 X. M. Feng, D. Huang, X. H. Lu, G. J. Feng, J. Xing, J. Lu, K. Xu, W. W. Xia, Y. Meng, T. Tao, L. R. Li and Z. F. Gu, Dev., Growth Differ., 2014, 56, 615-624.

18 F. M. Chen, R. Chen, X. J. Wang, H. H. Sun and Z. F. Wu, Biomaterials, 2009, 30, 5215-5224.

19 K. Whang, D. C. Tsai, E. K. Nam, M. Aitken, S. M. Sprague, P. K. Patel and K. E. Healy, J. Biomed. Mater. Res., 1998, 42, 491-499.

20 S. G. Wang, Q. Cai, J. W. Hou, J. Z. Bei, T. Zhang, J. Yang and Y. Q. Wan, J. Biomed. Mater. Res., Part A, 2003, 66, 522-531.

21 H. Shen, X. X. Hu, J. Z. Bei and S. G. Wang, Biomaterials, 2008, 29, 2388-2399.

22 H. Shen, X. X. Hu, F. Yang, J. Z. Bei and S. G. Wang, Biomaterials, 2011, 32, 3404-3412.

23 Z. Wang, L. Chen, Y. Wang, X. Chen and P. Zhang, ACS Appl. Mater. Interfaces, 2016, 8, 26559-26569.

24 H. Liu, W. L. Li, B. H. Luo, X. X. Chen, W. Wen and C. R. Zhou, Mater. Sci. Eng., C, 2017, 79, 399-409.

25 Y. Li, W. Yang, X. K. Li, X. Zhang, C. R. Wang, X. F. Meng, Y. F. Pei, X. L. Fan, P. H. Lan, C. H. Wang, X. J. Li and Z. Guo, ACS Appl. Mater. Interfaces, 2015, 7, 5715-5724.

26 X. M. Sun, L. Y. Cheng, J. W. Zhao, R. Jin, B. S. Sun, Y. M. Shi, L. Zhang, Y. G. Zhang and W. G. Cui, J. Mater. Chem. B, 2014, 2, 3636-3645.

27 E. Ko, K. Yang, J. Shin and S. W. Cho, Biomacromolecules, 2013, 14, 3202-3213.

28 H. Liu, W. L. Li, W. Wen, B. H. Luo, M. X. Liu, S. Ding and C. R. Zhou, Mater. Sci. Eng., C, 2017, 81, 280-290.

29 S. Jo, S. M. Kang, S. A. Park, W. D. Kim, J. Kwak and H. Lee, Macromol. Biosci., 2013, 13, 1389-1395.

30 S. Kim and C. B. Park, Langmuir, 2010, 26, 14730-14736.

31 J. M. Lane and H. S. Sandhu, Orthop. Clin. N. Am., 1987, 18, 213-225.

32 M. Y. Cheng, J. U. Deng, F. Yang, Y. D. Gong, N. M. Zhao and X. F. Zhang, Biomaterials, 2003, 24, 2871-2880. 
33 X. L. Zhu, W. G. Cui, X. H. Li and Y. Jin, Biomacromolecules, 2008, 9, 1795-1801.

34 S. Zhou, Q. Chang, F. Lu and M. Xing, Adv. Healthcare Mater., 2017, 6, 1700131.

35 J. Zhou, X. Guo, Q. Zheng, Y. Wu, F. Cui and B. Wu, Colloids Surf., B, 2017, 152, 124-132.

36 Y. Cui, Y. Liu, Y. Cui, X. Jing, P. Zhang and X. Chen, Acta Biomater., 2009, 5, 2680-2692.

37 H. A. Afshar and A. Ghaee, Carbohydr. Polym., 2016, 151, 1120-1131.

38 Y. W. Yang, G. Camporeale, E. Sardella, G. Dilecce, J. S. Wu, F. Palumbo and P. Favia, Plasma Processes Polym., 2014, 11, 1102-1111.

39 H. T. Cui, Y. Wang, L. G. Cui, P. B. Zhang, X. H. Wang, Y. Wei and X. S. Chen, Biomacromolecules, 2014, 15, 3146-3157.
40 N. H. Dormer, Y. Qiu, A. M. Lydick, N. D. Allen, N. Mohan, C. J. Berkland and M. S. Detamore, Tissue Eng., Part A, 2012, 18, 757-767.

41 J. Doorn, S. J. Roberts, J. Hilderink, N. Groen, A. van Apeldoorn, C. van Blitterswijk, J. Schrooten and J. de Boer, Tissue Eng., Part A, 2013, 19, 1817-1828.

42 M. Hughes-Fulford and C. F. Li, J. Orthop. Surg. Res., 2011, 6, 8.

43 M. Du, T. Zhu, X. Q. Duan, S. H. Ge, N. Li, Q. F. Sun and P. S. Yang, Mater. Sci. Eng., C, 2017, 70, 62-70.

44 N. Liu and Z. L. Wang, RSC Adv., 2016, 6, 50322-50330.

45 X. Y. Zhao, Y. Han, J. W. Li, B. Cai, H. Gao, W. Feng, S. Q. Li, J. G. Liu and D. S. Li, Mater. Sci. Eng., C, 2017, 78, 658-666. 